\title{
Correlations, compressibility, and capacitance in double-quantum-well systems in the quantum Hall regime
}

\author{
T. Jungwirth \\ Institute of Physics ASCR, Cukrovarnická 10, 16200 Praha 6, Czech Republic \\ A.H. MacDonald \\ Department of Physics, Indiana University, Bloomington, IN 47405
}

(Received September 22, 2018)

\begin{abstract}
In the quantum Hall regime, electronic correlations in double-layer two-dimensional electron systems are strong because the kinetic energy is quenched by Landau quantization. In this article we point out that these correlations are reflected in the way the partitioning of charge between the two-layers responds to a bias potential. We report on illustrative calculations based on an unrestricted Hartree-Fock approximation which allows for spontaneous inter-layer phase coherence. The possibility of studying inter-layer correlations by capacitive coupling to separately contacted two-dimensional layers is discussed in detail.
\end{abstract}

Typeset using REVTEX 


\section{INTRODUCTION}

Technological progress has made it possible to fabricate epitaxially grown semiconductor systems with nearby two-dimensional electron layers and has led to interest in the physics of the various inter-layer coupling effects which occur as a consequence. As shown in Figure 1, these systems consists of two parallel electron layers confined by narrow rectangular quantum wells. In standard GaAs/AlGaAs structures with the width of the wells of order $10 \mathrm{~nm}$ and the barrier height about $250 \mathrm{meV}$, electron wavefunctions are strongly localized around the center of each quantum well and the overlap between layers is very small. To date coupling effects have been observed primarily in the transport properties of double-layer systems. For example, inter-layer electron-electron interactions leadel 2 to frictional drag voltages when charge in one layer is moved relative to charge in the nearby layer. Inter-layer tunneling leads to quantum interference effects which are responsible for interesting dependence of both in-pland 3 and inter-pland ${ }^{1}$ conductances on the strength of a magnetic field oriented parallel to electron layers. In a strong perpendicular magnetic field, the kinetic energy of the electrons is quenched by Landau quantization and, at least in high-mobility systems, electron-electron interactions dominate the physics. For double-layer systems inter-layer interactions are responsible for novel broken symmetries 5 and, if tunneling between layers also occurs, for inordinate sensitivity to small tilts of the field away from the normal to the electron layers 6

In this paper we discuss the effect of inter-layer coupling on equilibrium properties of double-layer systems. In particular we consider the variation of the partitioning of charge between the two-layers as the total electron density is modified by adjusting an external

gate potential. Eisenstein and co-workers have measured this quantity for the case of more remotely spaced layers by combining a standard capacitive method with a measurement of the charge transferred between layers when the gate voltage is changed. Using the assumption (valid in that work) that inter-layer correlations could safely be neglected, they were able to relate the measured inter-layer current to the compressibility of the electron layer 
closest to the gate. In Section II we use an idealized model with infinitely narrow quantum wells to generalize their analysis to the case where inter-layer correlations are important. In a strong perpendicular magnetic field, the electronic properties of double-layer systems are extremely subtle. To date most studies 8 of double-layer systems have focused on systems with equal density in each layer. In this article we use an unrestricted Hartree-Fock approximation to obtain qualitative results as a function of layer separation over the full range of total filling factors and bias potentials in the quantum Hall regime. The Hartree-Fock approximation allows for spontaneous inter-layer phase coherence two different points of view in Sections III and IV. In Section V we present and discuss the results predicted for Eisenstein's double-layer capacitance measurement by the unrestricted Hartree-Fock approximation. Finally, we present our conclusions in Section VI.

\section{NARROW-WELL DOUBLE-LAYER MODEL}

In this section we assume that only the lowest energy subband is relevant in each quantum well and, for convenience, we take the two quantum wells to be identical. We further assume that each quantum well is sufficiently narrow that we can replace the charge density in each by a zero-thickness layer located at the center of the quantum well. With these assumptions it follows that for fixed external charges (assumed to reside away from the double layer system) the energy of the double-layer system is given up to an irrelevant constant by

$$
\frac{E}{A}=\frac{e^{2} d}{2 \epsilon}\left(N_{R}-N_{0}\right)^{2}+\varepsilon\left(N-N_{R}, N_{R}\right)
$$

where $A$ is the area of the system, $N_{R}$ and $N_{L}=N-N_{R}$ are the areal densities of electrons in the right and left layers, $N$ is the total electron density and $N_{0}$ is determined by external charges as discussed below. In Eq. (11) $\varepsilon\left(N_{L}, N_{R}\right)$ would be the energy per area of the double-layer system if neutralizing external charges were located in each layer of the double-layer system. This quantity is the conventional point of contact between electron gas theory and experiment. For a given configuration of external charge, the charge distribution is determined by minimizing the sum of $\varepsilon\left(N_{L}, N_{R}\right)$ and the electrostatic energy. The 
zero-thickness layers, with areal charge densities $e N_{L}$ and $e N_{R}$, yield discontinuities in the dependence of the electric field along the direction between layers (which we take to be the $\hat{z}$ direction) across each layer. We assume that any charges induced by variation of the gate voltage go entirely into the electron layers so the electric field $E_{0}$ at the right boundary of the double-layer in Figure 1 is independent on the voltage and enters the problem as an input parameter. From the Poisson equation we then obtain

$$
\begin{aligned}
& E_{1}=E_{0}-\frac{|e|}{\epsilon} N_{R} \equiv \frac{|e|}{\epsilon}\left(N_{0}-N_{R}\right) \\
& E_{2}=E_{0}-\frac{|e|}{\epsilon}\left(N_{R}+N_{L}\right) .
\end{aligned}
$$

$N_{0}$ is defined by this equation. Note that changing $E_{2}$ is equivalent to changing $N=N_{R}+N_{L}$. (See Figure 1.)

The double-layer capacitance technique of Eisenstein et al. measures $R_{E}$, the ratio of the electric field change between the electron layers to the electric field change between the gate and the nearest electron layer:

$$
R_{E} \equiv \frac{d E_{1}}{d E_{2}}=\frac{d N_{R}}{d N}
$$

Given $N, \quad N_{R}$ is determined by minimizing the total energy described in Eq. (11) yielding,

$$
\mu_{L}\left(N-N_{R}, N_{R}\right)=\mu_{R}\left(N-N_{R}, N_{R}\right)+\frac{e^{2} d}{\epsilon}\left(N_{R}-N_{0}\right)
$$

where

$$
\mu_{L}\left(N_{L}, N_{R}\right) \equiv \frac{\partial \varepsilon\left(N_{L}, N_{R}\right)}{\partial N_{L}}
$$

and $\mu_{R}\left(N_{L}, N_{R}\right)$ is defined similarly. $\mu_{L}\left(N_{L}, N_{R}\right)$ includes all contributions to the chemical potential for electrons in the left layer except for the contribution from the electrostatic potentials and would be the full chemical potential if, as in conventional electron gas literature, neutralizing positive charges in each layer were assumed. It follows from Eq. (4) that

$$
R_{E}=\frac{d_{L L}-d_{R L}}{d+d_{L L}+d_{R R}-d_{R L}-d_{L R}}
$$


where we have followed Eisenstein et al. introducingt a set of lengths defined by

$$
d_{A B}\left(N_{L}, N_{R}\right) \equiv \frac{\epsilon}{e^{2}} \frac{\partial \mu_{A}\left(N_{L}, N_{R}\right)}{\partial N_{B}}
$$

In Eq. (7) $A$ and $B$ are layer labels. When inter-layer electron-electron interactions can be neglected $d_{L R}=d_{R L}=0$ and

$$
d_{A A}\left(N_{A}\right)=\frac{\epsilon}{e^{2} \kappa_{A} N_{A}^{2}}
$$

where $\kappa_{A}$ is the compressibility of the electron system in layer $A$ with the usual convention of a neutralizing background. For non-interaction electrons and zero magnetic field $d_{A A}=$ $d_{E} \equiv a_{0}^{*} / 4$ is independent of the electron density in layer $A$; here $d_{E}$ is the length defined by Eisenstein et al. and $a_{0}^{*}=\hbar^{2} \epsilon / m^{*} e^{2}$ is the effective Bohr radius of the semiconductor. For GaAs $a_{0}^{*} \approx 10 \mathrm{~nm}$ so $d_{E} \approx 2.5 \mathrm{~nm}$. For non-interacting electrons in a strong magnetic field, $d_{A A}=0$ when a Landau level is partially filled and $d_{A A}=\infty$ at integer Landau level filling factor.

\section{UNRESTRICTED HARTREE-FOCK APPROXIMATION FOR INTERLAYER CORRELATIONS: SELF-CONSISTENT FIELD EQUATION APPROACH}

For decoupled layers, electron-electron interactions can reduce or event change the sign of $d_{A A}$. In the following sections we discuss the effect of inter-layer coupling on $R_{E}$. In the absence of a magnetic field inter-layer interactions have little effect 10 on $R_{E}$ at experimentally accessible layer separations. The situation is different at strong magnetic fields where the kinetic energy of the electrons is quenched and interaction effects are very strong. The problem of finding accurate results for the dependence of the ground state energy in this regime on the density in each layer and on the layer separation is a difficult one which is largely unsolved because perturbative approaches are unsuitable. Numerical exact-diagonalization results can provide guidance and some results 1 are already available using this approach. In the following sections we follow an alternate line by developing a simple Hartree-Fock 
approximation for biased double-layer systems. In our Hartree-Fock approximation interlayer correlations can be generated by forming broken-symmetry states with spontaneous inter-layer phase coherence, as we describe in more detail below. Such a broken symmetry does in fact $\mathbf{O}^{\mathrm{S}}$ occur in double-layer systems in strong magnetic fields, although not over as wide a range of densities and layer separations as in our calculations. The correlations which appear only in connection with a broken symmetries in the single Slater determinant states of the Hartree-Fock approximation are more generically associated with correlated quantum fluctuations in the electronic configuration. Nevertheless, we believe that the approximation for the energy of the double-layer system which is obtained in the Hartree-Fock approximation is meaningful and that our results will be helpful in the interpretation of double-layer capacitance studies.

We will assume that the electronic spins are fully polarized by the magnetic field and adopt a useful pseudospin language ism the total Hilbert space $\mathcal{H}$ is expressed as a direct product of the orbital Hilbert space $\mathcal{H}_{o}$ and pseudospin Hilbert space $\mathcal{H}_{s}$. Choosing $\phi_{0, m}(x, y)$, symmetric-gauge two-dimensional free particle eigenstates 11 in the lowest Landau level, as a basis of $\mathcal{H}_{o}$ and assuming zero layer thickness, the basis vectors of $\mathcal{H}$ can be written as

$$
\psi_{A, m}=\delta\left(z-z_{A}\right) \phi_{0, m}(x, y)
$$

where $z_{L}=0$ and $z_{R}=d$. Then the $\mathcal{H}_{s}$ spinors

$$
\left(\begin{array}{l}
1 \\
0
\end{array}\right),\left(\begin{array}{l}
0 \\
1
\end{array}\right)
$$

describe states in which an electron is localized in the left or right quantum well respectively. This language suggests a magnetic analogy for the double-layer system. For example, with the definition in Eq. (10) the $\hat{z}$-component of the total pseudospin operator $\vec{S}$ is proportional to the difference in density between the layers since $<S^{z}>=\left(N_{L}-N_{R}\right) / 2$. The $\hat{x}$ and $\hat{y}$ components of the pseudospin operators correspond to components of the density operator which are off diagonal in layer indices; non-zero expectation values are possible only 
when there is inter-layer phase coherence. In a special limit of $d=0$ interactions between electrons in the same layer are identical to those between electrons in different layers and the Hamiltonian has $S U(2)$ symmetry: $\left[H, S^{\mu}\right]=0$ and eigenstates occur in multiplets with pseudospin quantum number $S$ and degeneracy $2 S+1$. For finite layer separation only $S_{z}$ is a good quantum number.

In the limit of large $d$, the equilibrium charge distribution is determined solely by electrostatic considerations and the functions (9) describe eigenstates of the corresponding Hartree Hamiltonian. In this limit, it follows from Eq. (幽 that the two-layers can be brought into equilibrium only if $N_{R}=N_{0}$, i.e., only if the electric field between the layers is equal to zero. Any change in the gate voltage will result in a change in charge density exclusively in the left well. In the strong magnetic field limit considered here, equilibrium can be established only if the total filling factors $\nu \equiv 2 \pi \ell^{2} N \in\left(\nu_{0}, 1+\nu_{0}\right)$, where the threshold filling factor

$$
\nu_{0}=\frac{2 \pi l^{2} \epsilon}{|e|} E_{0}
$$

and the magnetic length $\ell \equiv(\hbar /|e| B)^{1 / 2}$. Outside this interval the left layer lowest Landau level is either empty or is completely filled. Therefore,

$$
\nu_{R}=\left\{\begin{array}{ll}
\nu & \text { for } \nu<\nu_{0} \\
\nu_{0} & \text { for } \nu_{0} \leq \nu \leq 1+\nu_{0} \\
\nu-1 & \text { for } \nu>1+\nu_{0}
\end{array} .\right.
$$

For smaller $d$ the charge distribution depends on intra and inter-layer correlations. In what follows we use dimensionless units expressing energy in units of $e^{2} / 4 \pi \epsilon \ell$ and lengths in units of the magnetic length, $\ell$. Deriving the Hartree-Fock self-consistent equation we will, for simplicity, neglect tunneling between the two layers. We will return to a discussion of the influence of tunneling later. We will assume that the translational symmetry within each two-dimensional layer is not broken so that the orbital degeneracy of the Landau levels is maintained.

In our Hartree-Fock calculations we do not require $S_{z}$ to be a good quantum number. Allowing this symmetry to be broken gives rise to a much better variational estimate of the 
ground state energy and results in states with spontaneous phase coherence between the layers. We seek eigenstates $|\Psi\rangle$ of the Hartree-Fock Hamiltonian with, generally, non-zero expectation value of the $\hat{x}$ and $\hat{y}$ components of the pseudospin operator. The general form of the two orthogonal pseudospinors for the lower ('-') and higher ('+') energy Landau levels are:

$$
\alpha_{-} \equiv\left(\begin{array}{c}
\left\langle\psi_{L} \mid \Psi_{-}\right\rangle \\
\left\langle\psi_{R} \mid \Psi_{-}\right\rangle
\end{array}\right)=\left(\begin{array}{c}
\cos \frac{\theta}{2} \\
\mathrm{e}^{i \varphi} \sin \frac{\theta}{2}
\end{array}\right)
$$

and

$$
\alpha_{+} \equiv\left(\begin{array}{c}
\left\langle\psi_{L} \mid \Psi_{+}\right\rangle \\
\left\langle\psi_{R} \mid \Psi_{+}\right\rangle
\end{array}\right)=\left(\begin{array}{c}
\sin \frac{\theta}{2} \\
-\mathrm{e}^{i \varphi} \cos \frac{\theta}{2}
\end{array}\right)
$$

The Hamiltonian in the pseudospin Hilbert space has a $2 \times 2$ matrix representation

$$
H=\left(\begin{array}{cc}
\varepsilon_{L} & 0 \\
0 & \varepsilon_{R}
\end{array}\right)+\left(\begin{array}{cc}
\Sigma_{L L} & \Sigma_{L R} \\
\Sigma_{R L} & \Sigma_{R R}
\end{array}\right),
$$

where the Hartree potential appears in $\varepsilon_{L}$ and $\varepsilon_{R}$ and $\Sigma_{i j}$ are matrix elements of the exchange self-energy. The orbital indices are omitted in Eq. (13-15) since the exchange self-energy is independent of the orbital quantum number of the Landau level, as we will explicitly prove. The self-consistent pseudospinor orientations, and consequently the charge distribution, can be determined by solving the Hartree-Fock equations iteratively using the expression for the self-energy given below.

\section{A. Total filling factor $\nu \leq 1$}

In case when the total filling factor $\nu \leq 1$ only the lower energy pseudospinor (13) is occupied and we obtain for the filling factor in the right layer

$$
\nu_{R}=\nu \sin ^{2} \frac{\theta}{2}
$$

Including the Hartree self-energy and choosing the zero of energy so that $\varepsilon_{R}=0$ it follows directly from the Poisson that 


$$
\varepsilon_{L}=2 d\left(\nu_{0}-\nu \sin ^{2} \frac{\theta}{2}\right) .
$$

The evaluation of the exchange self-energy is more cumbersome; we describe the derivation in detail for $\Sigma_{L L}$. The same procedure can be directly applied to all other self-energy matrix elements. Assuming that for the low-energy pseudospinor all orbital states are occupied with probability $\nu$ we find that

$$
\begin{aligned}
\Sigma_{L L}\left(m, m^{\prime}\right) & =-\nu \sum_{n} \int \mathrm{d} \vec{r} \psi_{L, m}^{*}\left(\vec{r}_{1}\right) \Psi_{-, n}^{*}\left(\vec{r}_{2}\right) \times \\
& \times \Psi_{-, n}\left(\vec{r}_{1}\right) \psi_{L, m^{\prime}}\left(\vec{r}_{2}\right) V\left(\vec{r}_{1}-\vec{r}_{2}\right) .
\end{aligned}
$$

The fractional occupation results from taking the zero temperature limit of a finitetemperature Hartree-Fock expressions and occurs because of the Landau level degeneracy of the Hartree-Fock eigenvalues. Using (9), (13) and performing a Fourier transformation of the Coulomb potential $V\left(\vec{r}_{1}-\vec{r}_{2}\right)$, Eq. (18) can be rewritten as

$$
\begin{aligned}
\Sigma_{L L}\left(m, m^{\prime}\right) & =-\nu \cos ^{2} \frac{\theta}{2} \sum_{n} \int \mathrm{d}^{2} r_{\perp} \int \frac{\mathrm{d}^{2} q_{\perp}}{(2 \pi)^{2}} V_{e f f}\left(\vec{q}_{\perp}\right) \times \\
& \times \phi_{0, m}^{*}\left(\vec{r}_{\perp 1}\right) \mathrm{e}^{i \vec{q}_{\perp} \vec{r}_{\perp 1}} \phi_{0, n}\left(\vec{r}_{\perp 1}\right) \phi_{0, n}^{*}\left(\vec{r}_{\perp 2}\right) \mathrm{e}^{-i \vec{q}_{\perp} \vec{r}_{\perp 2}} \phi_{0, m^{\prime}}\left(\vec{r}_{\perp 2}\right)
\end{aligned}
$$

where

$$
\begin{aligned}
V_{e f f}\left(\vec{q}_{\perp}\right) & =\frac{e^{2}}{\epsilon} \int \mathrm{d} z \int \frac{d q_{z}}{2 \pi} \delta\left(z_{1}\right) \delta\left(z_{2}\right) \frac{\mathrm{e}^{i q_{z}\left(z_{1}-z_{2}\right)}}{q_{\perp}^{2}+q_{z}^{2}} \\
& =\frac{e^{2}}{2 \epsilon\left|q_{\perp}\right|} .
\end{aligned}
$$

The sum over $n$ in Eq. (19) can be evaluated analytically as shown in the Appendix, and is proportional to $\delta_{m^{\prime}, m}$. Thus, the exchange self-energy is diagonal and independent of $m$ and every state in the Landau level has the same spinor as anticipated. Finally we obtain

$$
\Sigma_{L L}=-\nu \cos ^{2} \frac{\theta}{2} I_{A}
$$

where for the case of Coulomb interactions the intra-layer exchange integral $I_{A}=\sqrt{\pi / 2}$. 
A similar calculation shows that $\Sigma_{R R}$ is given by the same expression with $\cos ^{2} \theta / 2$ replaced by $\sin ^{2} \theta / 2$. For the inter-layer exchange self-energies, the potential $V_{\text {eff }}$ is modified because of the layer separation $d$. For Coulomb interactions the inter-layer exchange integral is

$$
I_{E}=\int_{0}^{\infty} \mathrm{d} q \exp \left(-\frac{q^{2}}{2}-d q\right)
$$

Using the explicit expressions for the Hartree and exchange self-energies derived above in Eq. (15) we obtain the Hamiltonian

$$
\begin{aligned}
& H=\left(\begin{array}{cc}
2 d\left(\nu_{0}-\nu \sin ^{2} \frac{\theta}{2}\right) & 0 \\
0 & 0
\end{array}\right)- \\
& -\nu\left(\begin{array}{cc}
\cos ^{2} \frac{\theta}{2} I_{A} & \sin \frac{\theta}{2} \cos \frac{\theta}{2} \mathrm{e}^{i \varphi} I_{E} \\
\sin \frac{\theta}{2} \cos \frac{\theta}{2} \mathrm{e}^{-i \varphi} I_{E} & \sin ^{2} \frac{\theta}{2} I_{A}
\end{array}\right) .
\end{aligned}
$$

The eigenfunctions of this Hamiltonian are easily found by expanding it in terms of Pauli spin matrices:

$$
H=H_{0}+\overrightarrow{\mathcal{B}} \vec{\sigma}
$$

where

$$
H_{0}=\frac{\varepsilon_{L}}{2}-\frac{\nu}{2} I_{A}
$$

and the effective 'Zeeman' field $\overrightarrow{\mathcal{B}}$ has components

$$
\begin{aligned}
\mathcal{B}_{x} & =-\frac{\nu}{2} \sin \theta \cos \varphi I_{E} \\
\mathcal{B}_{y} & =-\frac{\nu}{2} \sin \theta \sin \varphi I_{E} \\
\mathcal{B}_{z} & =\frac{\varepsilon_{L}}{2}-\frac{\nu}{2} \cos \theta I_{A} .
\end{aligned}
$$


The low energy eigenspinor of $H$ will be the spinor which is aligned with $\overrightarrow{\mathcal{B}}$. Self-consistency is therefore achieved when $\overrightarrow{\mathcal{B}}$ has the same orientation as the spinor from which the exchange self-energy was constructed. This condition reduces to an algebraic equation for the polar angle $\theta$ :

$$
\tan \theta=\frac{\nu \sin \theta I_{E}}{\nu \cos \theta I_{A}-\epsilon_{L}}
$$

If $\theta \neq 0, \pi$, exchange electron-electron interactions lead to phase coherence between electrons in different layers. The direction of the ground state pseudospin is specified by the angles $\theta$ and $\varphi$. Note that the azimuthal angle $\varphi$ is arbitrary.

\section{B. Total filling factor $\nu>1$}

At $\nu>1$ all states in the low energy Landau level are full and the high energy Landau level is partially occupied. The contribution of the higher energy Landau level to both Hartree and exchange self-energies has to be included. For example, the filling factor in the right layer for $\nu>1$ is given by

$$
\nu_{R}=\sin ^{2} \frac{\theta}{2}+(\nu-1) \cos ^{2} \frac{\theta}{2} .
$$

We again obtain degenerate Landau levels. In this case we find that the pseudospinor Hamiltonian is given by

$$
\begin{aligned}
H & =\left(\begin{array}{cc}
2 d\left(\nu_{0}-\sin ^{2} \frac{\theta}{2}-(\nu-1) \cos ^{2} \frac{\theta}{2}\right) & 0 \\
0 & 0
\end{array}\right)- \\
& -\left(\begin{array}{cc}
\cos ^{2} \frac{\theta}{2} I_{A} & \sin \frac{\theta}{2} \cos \frac{\theta}{2} \mathrm{e}^{i \varphi} I_{E} \\
\sin \frac{\theta}{2} \cos \frac{\theta}{2} \mathrm{e}^{-i \varphi} I_{E} & \sin ^{2} \frac{\theta}{2} I_{A}
\end{array}\right)-
\end{aligned}
$$




$$
-(\nu-1)\left(\begin{array}{cc}
\sin ^{2} \frac{\theta}{2} I_{A} & -\sin \frac{\theta}{2} \cos \frac{\theta}{2} \mathrm{e}^{i \varphi} I_{E} \\
-\sin \frac{\theta}{2} \cos \frac{\theta}{2} \mathrm{e}^{-i \varphi} I_{E} & \cos ^{2} \frac{\theta}{2} I_{A}
\end{array}\right) .
$$

When this is expanded in terms of Pauli spin matrices it results in a effective Zeeman field given by

$$
\begin{aligned}
\mathcal{B}_{x} & =-\frac{2-\nu}{2} \sin \theta \cos \varphi I_{E} \\
\mathcal{B}_{y} & =-\frac{2-\nu}{2} \sin \theta \sin \varphi I_{E} \\
\mathcal{B}_{z} & =\frac{\varepsilon_{L}}{2}-\frac{2-\nu}{2} \cos \theta I_{A} .
\end{aligned}
$$

\section{UNRESTRICTED HARTREE FOCK APPROXIMATION: TOTAL ENERGY}

Eq. (27) often has more than one solution. The best unrestricted Hartree-Fock approximation to the ground state of the double-layer system is the solution with the lowest energy. In the Hartree-Fock approximation the total energy $E_{T O T}$ for two-dimensional electron systems in the strong magnetic field limit can be separated into electrostatic (Hartree) and exchange contributions. (The quantized kinetic energy is absorbed into the zero of energy and correlation effects are neglected in the Hartree-Fock approximation.) For a given $\nu$ constant the Hartree energy is (up to an arbitrary constant) proportional to the energy density in the intra-layer electric field:

$$
E_{H}=\frac{\epsilon d A E_{1}^{2}}{2}
$$

The electric field $E_{1}$ can be expressed as a function of pseudospin orientation using Eqs. (2), (11),(16), and (28). Using the dimensionless variables introduced in section II,

$$
\frac{E_{H}}{A}=\left\{\begin{array}{ll}
\frac{d\left(\nu_{0}-\nu \sin \frac{\theta}{2}\right)^{2}}{2 \pi} & \text { for } \nu \leq 1 \\
\frac{d\left(\nu_{0}-\sin ^{2} \frac{\theta}{2}-(\nu-1) \cos ^{2} \frac{\theta}{2}\right)^{2}}{2 \pi} & \text { for } \nu>1
\end{array} .\right.
$$


In evaluating the exchange energy it is necessary to avoid double-counting electron-electron interactions. For $\nu \leq 1$ only the low-energy pseudospinor is occupied while for $\nu>1$ both spinors are occupied and we find that

$$
\frac{E_{X}}{N A}= \begin{cases}\frac{1}{2} \alpha_{-}^{\dagger} H_{X} \alpha_{-} & \text {for } \nu \leq 1 \\ \frac{1}{2 \nu}\left(\alpha_{-}^{\dagger} H_{X} \alpha_{-}+(\nu-1) \alpha_{+}^{\dagger} H_{X} \alpha_{+}\right) & \text {for } \nu>1\end{cases}
$$

where $H_{X}$ is the exchange contribution to the Hartree-Fock Hamiltonian. (Explicit expressions for $H_{X}$ were derived for both $\nu \leq 1$ and $\nu>1$ in the previous section.) Using Eqs. (23),(29),(33) and the definition of the filling factor we obtain the following results, in dimensionless units, for the dependence of the exchange energy on pseudospin orientation. For $\nu \leq 1$

$$
\frac{E_{X}}{A}=-\frac{\nu^{2}}{4 \pi}\left(I_{A}\left(\sin ^{4} \frac{\theta}{2}+\cos ^{4} \frac{\theta}{2}\right)+2 I_{E} \sin ^{2} \frac{\theta}{2} \cos ^{2} \frac{\theta}{2}\right)
$$

and for $\nu>1$

$$
\begin{aligned}
\frac{E_{X}}{A} & =-\frac{1}{4 \pi}\left(I_{A}\left(\sin ^{4} \frac{\theta}{2}+\cos ^{4} \frac{\theta}{2}\right)\left(1+(\nu-1)^{2}\right)+\right. \\
& \left.+2 \sin ^{2} \frac{\theta}{2} \cos ^{2} \frac{\theta}{2}\left(I_{A}(\nu-1)+I_{E}(2-\nu)^{2}\right)\right) .
\end{aligned}
$$

Note, that minimizing the total energy with respect to the angle $\theta$, i.e., solving the equation

$$
\frac{\mathrm{d} E_{T O T} / A}{\mathrm{~d} \theta}=\frac{\mathrm{d}\left(E_{H}+E_{X}\right) / A}{\mathrm{~d} \theta}=0,
$$

yields an equation identical to that resulting from requiring the pseudospinor to selfconsistently solve Eq. (27). If more than one solution occurs we choose the solution with the lowest energy.

\section{NUMERICAL RESULTS}

We find that solutions to Eq. (36) can occur at $\theta=0$, at $\theta=\pi$ and at most at one $\theta \in(0, \pi) . \theta=0$ solutions correspond, for $\nu<1$, to all the electrons being in the left well, 
while $\theta=\pi$ solutions correspond, for $\nu<1$, to all electrons being in the right well. For $\nu>1$ these two solutions correspond to the full Landau levels in the left and right well respectively. For $\theta \in(0, \pi)$ both layers are partially occupied and in equilibrium. These solutions occur when

$$
\cos \theta=\left\{\begin{array}{ll}
\frac{d\left(2 \nu_{0}-\nu\right)}{\nu\left(I_{A}-I_{E}-d\right)} & \text { for } \nu \leq 1 \\
\frac{d\left(2 \nu_{0}-\nu\right)}{(2-\nu)\left(I_{A}-I_{E}-d\right)} & \text { for } \nu>1
\end{array} .\right.
$$

From Eq. (37) we see that both layers can be partially occupied only in the region of the $\nu-d$ plane where the absolute value of the right-hand side of Eq. (37) is less than 1. (Recall that $I_{E}$ has a dependence on $d$ which is implicit in these equations.) For $0 \leq \nu \leq 1$ the boundary of this region is defined by the curves

$$
d=\left\{\begin{array}{ll}
\frac{\nu}{\nu-\nu_{0}} \frac{I_{A}-I_{E}}{2} & \text { for } \nu \leq 2 \nu_{0} \\
\frac{\nu}{\nu_{0}} \frac{I_{A}-I_{E}}{2} & \text { for } \nu>2 \nu_{0}
\end{array},\right.
$$

while for $1 \leq \nu \leq 2$ the boundary is defined by the curves

$$
d=\left\{\begin{array}{ll}
\frac{2-\nu}{1-\nu_{0}} \frac{I_{A}-I_{E}}{2} & \text { for } \nu \leq 2 \nu_{0} \\
\frac{2-\nu}{1-\nu+\nu_{0}} \frac{I_{A}-I_{E}}{2} & \text { for } \nu>2 \nu_{0}
\end{array} .\right.
$$

The solution with the two layers in equilibrium is always lowest in energy whenever it is self-consistent, i.e. whenever a local energy minimum occurs for $\theta \in(0, \pi)$. When this solution doesn't exist, the polar angle $\theta=0$ for $\nu>2 \nu_{0}$ and $\theta=\pi$ for $\nu<2 \nu_{0}$. In the cases $\nu_{0} \leq 0$ or $\nu_{0} \geq 1 \theta=0$ or $\theta=\pi$ throughout the strong magnetic field regime. In Figure 2, Figure 3 and Figure 6 we show results obtained at $\nu_{0}=1 / 4, \nu_{0}=1 / 2$ and $\nu_{0}=3 / 4$ when there is no inter-layer hopping. The upper panel of each figure is a phase diagram which shows the state of the system as a function of layer separation and total filling factor. Note that there is a mirror symmetry along the line $\nu=1$ between the phase diagrams for $\nu_{0}=x$ $(x<1 / 2)$ and $\nu_{0}=1-x$. In Region I in these phase diagrams the two layers are not in 
equilibrium. In the left Region I all the electrons are in the right layer and the Hartree-Fock eigenenergy for the left layer lies above the chemical potential. In the right Region I the left Landau level is completely filled and its Hartree-Fock eigenenergy lies below the Fermi energy. In Region II, $\theta \in(0, \pi)$, and each Hartree-Fock eigenfunction is a coherent linear combination of states localized in the two wells. We do not believe that this spontaneous phase coherence exists throughout the the entire Region II as indicated schematically by the dashed lines in Region II. For example, for the case $\nu_{0}=1 / 2, \nu=1$, which has been studied extensively both theoretically, 5, 12,13 and experimentally, 6, 14 spontaneous coherence is expected to occur only for $d<\approx 2$. It is very difficult to predict theoretically where, within Region II, spontaneous phase coherence will occur; the dashed lines in the figures are intended to suggest only that it is most likely near $\nu=1$ and at small layer separations where the Hartree-Fock approximation is most reliable. We believe that this question is best addressed experimentally. Stimulating such experiments is part of the motivation for this work.

The middle panels in Figure 2, Figure 3, and Figure 4 shows the optimal (self-consistent) filling factor in the right well as a function of the total filling factor for $d=1, d=5$, and $d \rightarrow \infty$. These three layer separations correspond to strongly coupled layers, weakly coupled layers and decoupled layers. For $d \rightarrow \infty$ all the charge goes into the right layer until the electric field reaches zero between the layers. When this point is reached all the incremental charge goes to the left layer until its Landau level is filled. Only then does the filling of the right layer resume. Exchange tends to favor unequal layer occupations except at the point where the layers are balanced, $\nu=2 \nu_{0}$, so that the left-layer is not occupied until larger total filling factors at smaller $d$. Once the occupation of the left layer begins, the right layer occupation gradually decreases as the left layer Landau level is filled.

The bottom panels of Figure 2 , Figure 3, and Figure 1 show the dependence of the Eisenstein ratio $R_{E}$ on total filling factor. In Region II both layers are in equilibrium and Eqs. (68) apply. From Eqs. (34) and (35) we obtain the Hartree-Fock values for the length parameters 


$$
\begin{aligned}
& d_{L L}=d_{R R}=-\frac{I_{A}}{2} \\
& d_{L R}=d_{R L}=-\frac{I_{E}}{2}
\end{aligned}
$$

and the Hartree-Fock Eisenstein ratio reads

$$
R_{E}=-\frac{I_{A}-I_{E}}{2\left(d-I_{A}+I_{E}\right)} .
$$

For large layer separations $d \gg I_{A}$ and inter-layer coupling can be neglected so that $R_{E}$ is proportional to the reciprocal value to the compressibility of an individual 2D layer. For the Coulomb interaction the Hartree-Fock theory in this limit gives $R_{E}=-I_{A} / 2 d=$ $-\sqrt{\pi / 8} / d$, missing the anomaliest associated with incompressible fractional quantum Hall states seen experimentally. At smaller $d$ the electrostatic term in the denominator becomes less dominant and inter-layer interactions become important. For small $d, I_{A}-I_{E}=$ $d-d^{2} \sqrt{\pi / 8}+\cdots$ so that in this limit the Hartree-Fock theory gives $R_{E}=-\sqrt{2 / \pi} / d$, diverging for $d \rightarrow 0$. The Hartree-Fock Eisenstein ratio within Region II is a negative monotonically increasing function of $d$ for all $d \in(0, \infty)$, as shown in Figure 5 . Neglecting the inter-layer interactions yields an unphysical divergence of $R_{E}$ at $d=I_{A}$.

In the discussion of equilibrium properties of the double-layer electron system presented above, tunneling between the 2D layers was neglected. In a tight-binding model, the tunneling contribution to the Hartree-Fock Hamiltonian is

$$
H_{t}=\left(\begin{array}{ll}
0 & t \\
t & 0
\end{array}\right)
$$

where $t$ is a phenomenological parameter which is in practise chosen to match either experimental or calculated values of the splitting between the two-lowest subbands of the double-layer system. The self-consistent procedure derived for $t=0$, is readily generalized to include this term in the Hamiltonian. We find that for $t \neq 0$ both layers are partially filled and in equilibrium throughout the strong magnetic field regime. The tunneling term in the Hamiltonian favors equal layer densities and therefore competes with the exchange electron-electron interactions. The filling factor $\nu_{R}$ and the Eisenstein ratio $R_{E}$ as a function 
of $\nu$ are shown in Figure 6 for several values of $t$ and for $\nu_{0}=1 / 2$. Note that the steps in $R_{E}$ associated with establishing equilibrium between the two layers are smeared by tunneling.

\section{CONCLUSIONS}

In this article we have shown how electron-electron interactions beyond a simple electrostatic approximation influence the dependence on a remote gate voltage of the partitioning of electric charge in a double-layer system. Our calculations are based on an unrestricted Hartree-Fock approximation which can introduce inter-layer correlations by forming a broken symmetry state with spontaneous inter-layer phase coherence. We have made contact with potential experiments by expressing our results in terms of the Eisenstein ratio, which is proportional to the rate of charge transfer between layers when the gate voltage is varied. Our calculations demonstrates the essential role of inter-layer correlations; if they were neglected in our calculations the Eisenstein ratio would have an unphysical divergence at

$d=\sqrt{\pi / 2}$. The Hartree-Fock approximation we use has deficiencies that are known to be important in this system. In particular, it does not capture the anomalies in the Eisenstein ratio which are associated with the fractional quantum Hall effect. However, we believe that our calculation provides a useful qualitative picture which will be helpful in guiding and interpreting experimental studies of coupled double-layer electron systems.

\section{ACKNOWLEDGMENTS}

We acknowledge helpful interactions with J.P. Eisenstein. This work has been supported by the National Science Foundation through grants NSF INT-9106888 and DMR-9416906.

\section{APPENDIX}

The sum over $n$ in Eq. (19) can be calculated using known identities for symmetricgauge eigenfunctions. It is useful to introduce a factor $G_{i, j}(k)$ ( $G$ is a function of complex 
wavevector $\left.k=k_{x}+i k_{y}\right)$ defined as

$$
G_{i, j}(k)=\left(\frac{j !}{i !}\right)^{1 / 2}\left(\frac{-i k}{\sqrt{2}}\right)^{i-j} L_{j}^{i-j}\left(\frac{k \bar{k}}{2}\right),
$$

where $L_{j}^{i-j}\left(\frac{k \bar{k}}{2}\right)$ is the generalized Laguerre polynomial. The relation between $G$ and matrix elements of $\exp (i \vec{k} \cdot \vec{r})$ reads

$$
\int d^{2} r \phi_{0, i}^{*}(r) \mathrm{e}^{i \vec{k} \cdot \vec{r}} \phi_{0, j}(r)=\mathrm{e}^{\frac{-|k|^{2}}{2}} G_{i, j}(k l)
$$

Then, since

$$
\sum_{n} G_{i, n}\left(k_{1}\right) G_{n, j}\left(k_{2}\right)=\mathrm{e}^{\frac{-\bar{k}_{1} k_{2}}{2}} G_{i, j}\left(k_{1}+k_{2}\right)
$$

we obtain

$$
\sum_{n} \phi_{0, m}^{*}\left(\vec{r}_{\perp 1}\right) \mathrm{e}^{i \vec{q}_{\perp} \vec{r}_{\perp 1}} \phi_{0, n}\left(\vec{r}_{\perp 1}\right) \phi_{0, n}^{*}\left(\vec{r}_{\perp 2}\right) \mathrm{e}^{-i \vec{q}_{\perp} \vec{r}_{\perp 2}} \phi_{0, m^{\prime}}\left(\vec{r}_{\perp 2}\right)=\delta_{m, m^{\prime}} \exp \left(-q_{\perp}^{2}\right)
$$




\section{REFERENCES}

${ }^{1}$ T.J. Gramila, J.P. Eisenstein, A.H. MacDonald, L.N. Pfeiffer, and K.W. West, Phys. Rev. Lett. 66, 1216 (1991); Phys. Rev. B 47, 12957 (1993); U. Sivan, P.M. Solomon, and H. Shtrikman, Phys. Rev. Lett. 68, 1196 (1992).

2 P.J. Price, Physica B 117, 750 (1983); H.C. Tso, P. Vasilopoulos, and F.M. Peeters, Phys. Rev. Lett. 68, 2516 (1992); A.-P. Jauho, and H. Smith, Phys. Rev. B 47, 4420 (1993); L. Zheng, and A.H. MacDonald, Phys. Rev. B 48, 8203 (1993); K. Flensberg and Ben Yu-Kuang Hu, Phys. Rev. Lett. 73, 3572 (1994).

${ }^{3}$ J.A. Simmons, S.K. Lyo, N.E. Harff, and J.F. Klem, Phys. Rev. Lett. 73, 2256 (1994); S.K. Lyo, Phys. Rev. B 50, 4965 (1994); G. S. Boebinger, A. Passner, L. N. Pfeiffer, and K. W. West, Phys. Rev. B 43, 12673 (1990); J. Hu and A.H. MacDonald, Phys. Rev. B 46, 12554 (1992); Y. Berk, A. Kamenv, A. Palevksi, L.N. Pfeiffer, and K.W. West, Phys. Rev. B 51, 2604 (1995).

${ }^{4}$ J.P. Eisenstein et. al., Phys. Rev. B 44, 6511 (1991); J.A. Simmons et. al. Phys. Rev. B 47, 15741 (1993); S.K. Lyo and J.A. Simmons, J. Phys. Condens. Matter 5, L299 (1993).

${ }^{5}$ See for example Kun Yang, K. Moon, L. Zheng, A.H. MacDonald, S.M. Girvin, D. Yoshioka, and Shou-Cheng Zhang, Phys. Rev. Lett. 72, 732, 1994 and work cited therein.

${ }^{6}$ S.Q. Murphy, J.P. Eisenstein, G.S. Boebinger, L.N. Pfeiffer, and K.W. West, Phys. Rev. Lett. 72, 728 (1994); A.H. MacDonald, P.M. Platzman, and G.S. Boebinger, Phys. Rev. Lett. 65, 775 (1990); G.S. Boebinger et. al., Phys. Rev. Lett. 64, 1793 (1990); Y.W. Suen et. al., Phys. Rev. B 44, 5947 (1991).

${ }^{7}$ J.P. Eisenstein, L.N. Pfeiffer, and K.W. West, Phys. Rev. B 50, 1760 (1994); J.P. Eisenstein, L.N. Pfeiffer, and K.W. West, Phys. Rev. Lett. 68, 674 (1992).

${ }^{8}$ Reliable calculations of the ground state energy are possible using exact diagonalization for finite-size systems. See for example Chakraborty and P. Pietiläinen, Phys. Rev. Lett., 
59, 2784 (1987); E.H. Rezayi and F.D.M. Haldane, Bull. Am. Phys. Soc. 32, 892 (1987); Song He, S. Das Sarma and X.C. Xie, Phys. Rev. B 47, 4394 (1993); D. Yoshioka, A.H. MacDonald, and S.M. Girvin, Phys. Rev. B 39, 1932 (1989).

${ }^{9}$ For a brief review of the fractional quantum Hall effect in double-layer systems see A.H. MacDonald, Surface Science 229, 1 (1990).

${ }^{10}$ L. Zheng and A.H. MacDonald, Phys. Rev. B 49, 5522 (1994) and related unpublished calculations.

${ }^{11}$ See for example, A.H. MacDonald, Introduction to the Physics of the Quantum Hall Regime Proceedings of the 1994 Les Houches Summer School on Mesoscopic Physics, to be published by North Holland. Indiana University Preprint IUCM-94014.

${ }^{12}$ X.G. Wen and A. Zee, Phys. Rev. Lett. 69, 1811 (1992); X.G. Wen and A. Zee, Phys. Rev. B 47, 2265 (1993).

13 Z.F. Ezawa and A. Iwazaki, Int. J. of Mod. Phys. B, 19, 3205 (1992); Z.F. Ezawa and A. Iwazaki, Phys. Rev. B 47, 7295 (1993); Z.F. Ezawa, A. Iwazaki, Phys. Rev. B 48, 15189 (1993).

14 T.S. Lay, Y. W. Suen, H.C. Manoharan, X. Ying, M.B. Santos, and M. Shayegan, Phys. Rev. B 50, 17725 (1994). 


\section{FIGURES}

FIG. 1. Simplified band diagram for a gated double-quantum-well structure in a strong perpendicular magnetic field.

FIG. 2. Results for the threshold filling factor $\nu_{0}=1 / 4$ (no inter-layer hopping): a) Hartree-Fock phase diagram. b) Filling factor of the right quantum well as a function of the total filling factor for $d=1,5, \infty$. c) Eisenstein ratio as a function of the total filling factor for the same layer separations as in b).

FIG. 3. Results for the threshold filling factor $\nu_{0}=1 / 2$ (no inter-layer hopping): a) Hartree-Fock phase diagram. b) Filling factor of the right quantum well as a function of the total filling factor for $d=1,5, \infty$. c) Eisenstein ratio as a function of the total filling factor for the same layer separations as in b).

FIG. 4. Results for the threshold filling factor $\nu_{0}=3 / 4$ (no inter-layer hopping): a) Hartree-Fock phase diagram. b) Filling factor of the right quantum well as a function of the total filling factor for $d=1,5, \infty$. c) Eisenstein ratio as a function of the total filling factor for the same layer separations as in b).

FIG. 5. Eisenstein ratio in Region II as a function of the layer separation with inter-layer interaction taken into account (solid line) and for $I_{E}=0$ (dotted line).

FIG. 6. Results for the threshold filling factor $\nu_{0}=1 / 2$, for layer separation $d=1$ and for selected values of the inter-layer hopping parameter $t$ : a) Filling factor of the right quantum well as a function of the total filling factor. b) Eisenstein ratio as a function of the total filling factor. 


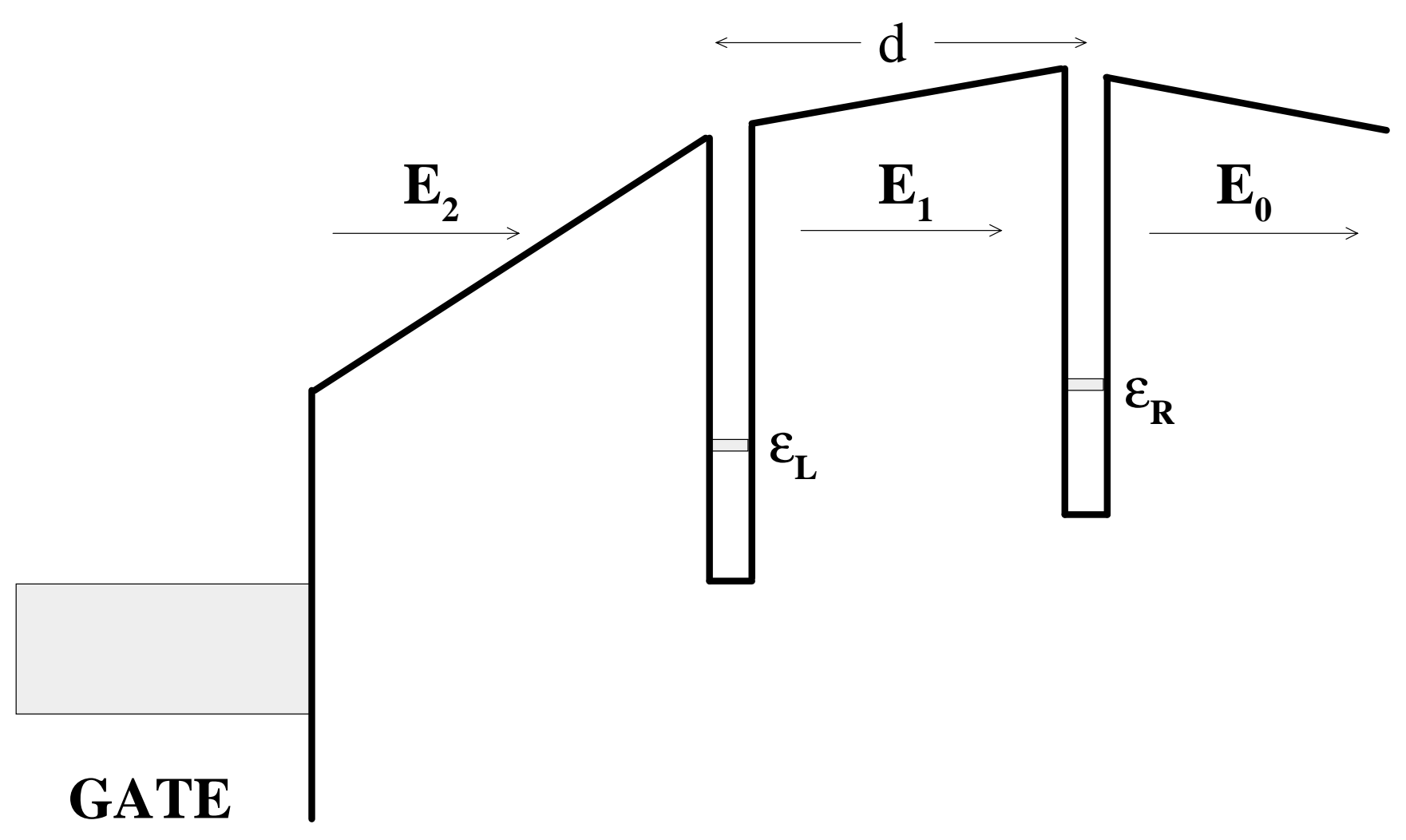




$$
v_{0}=1 / 4
$$

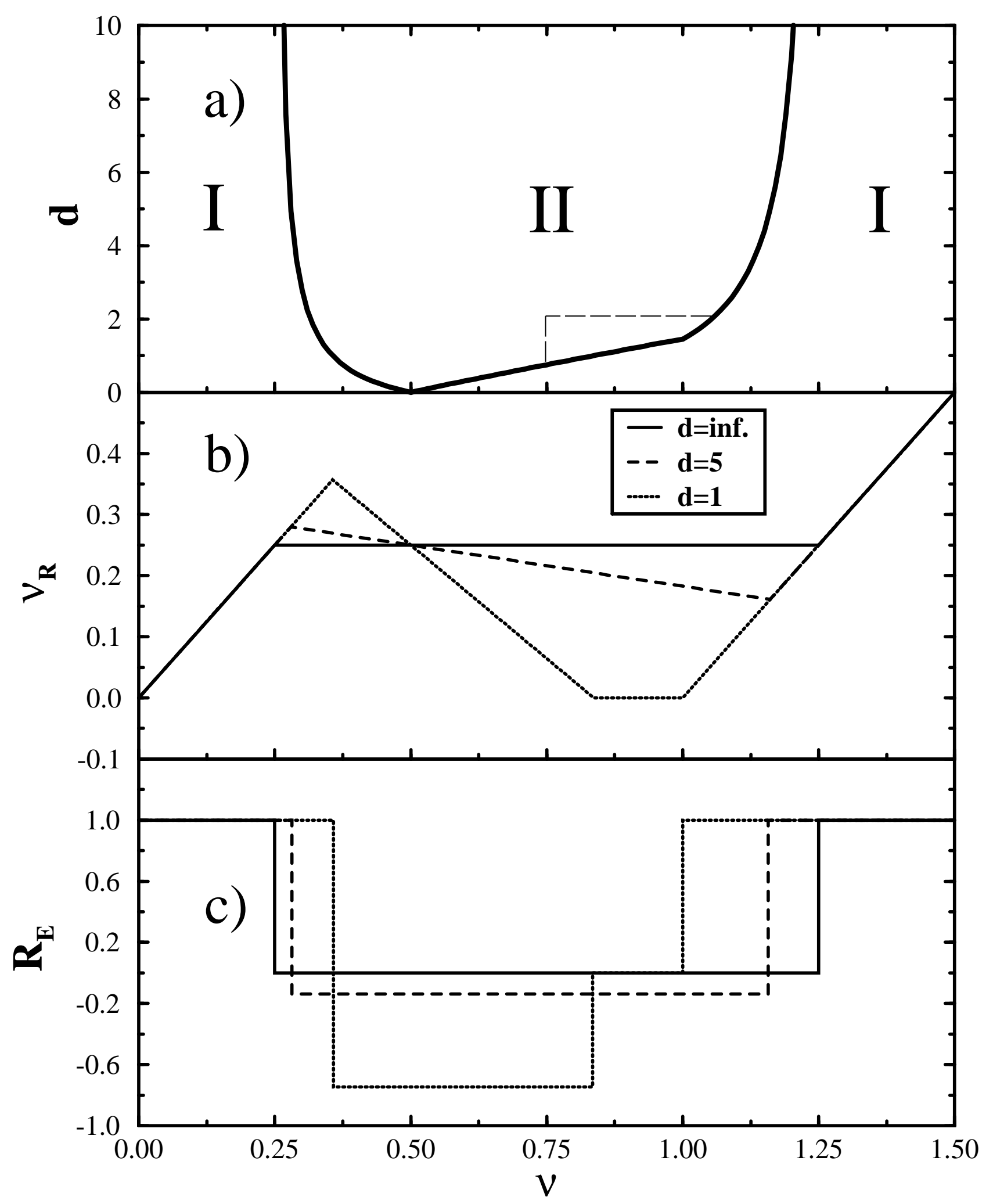




$$
v_{0}=1 / 2
$$

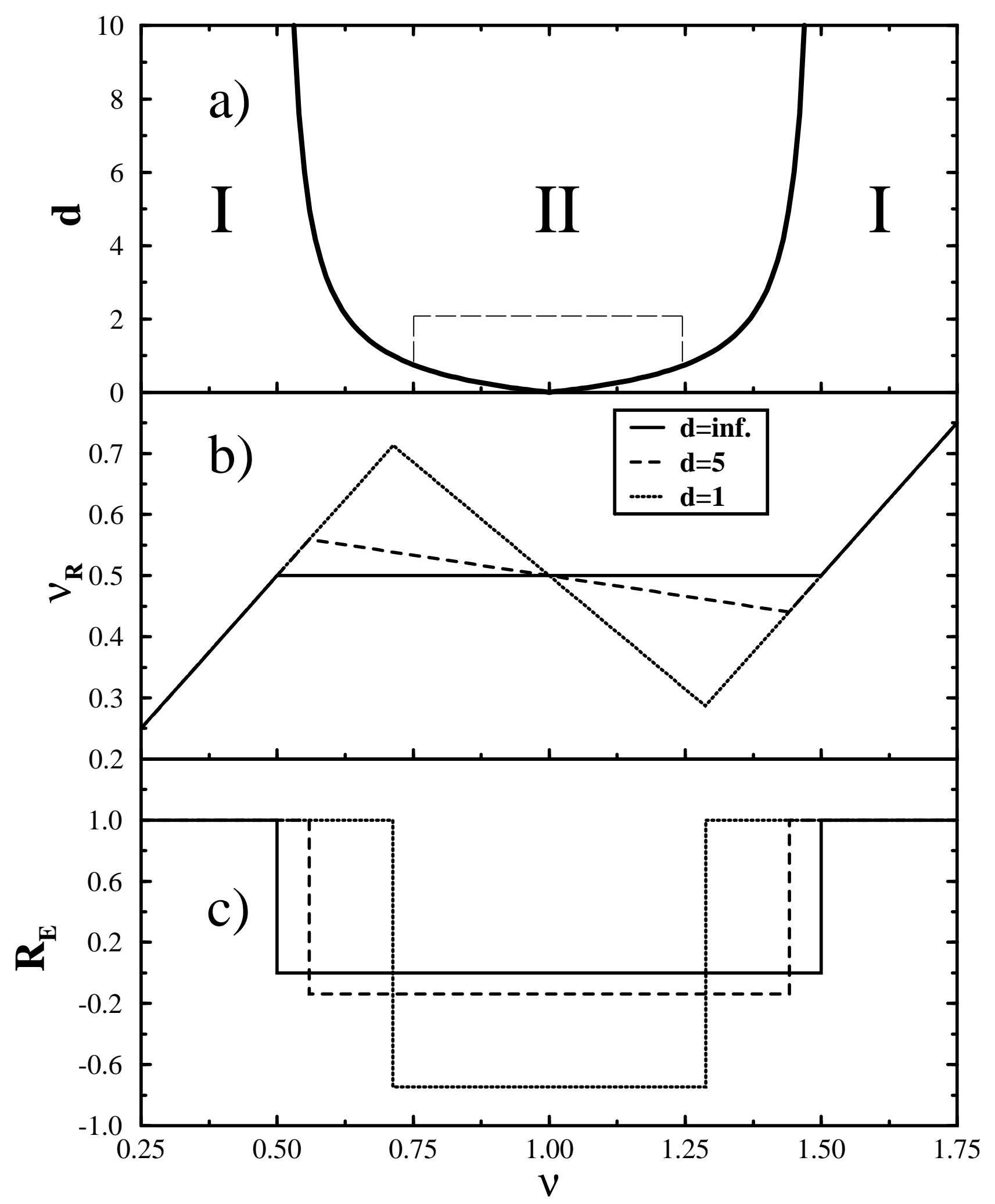




\section{$v_{0}=3 / 4$}

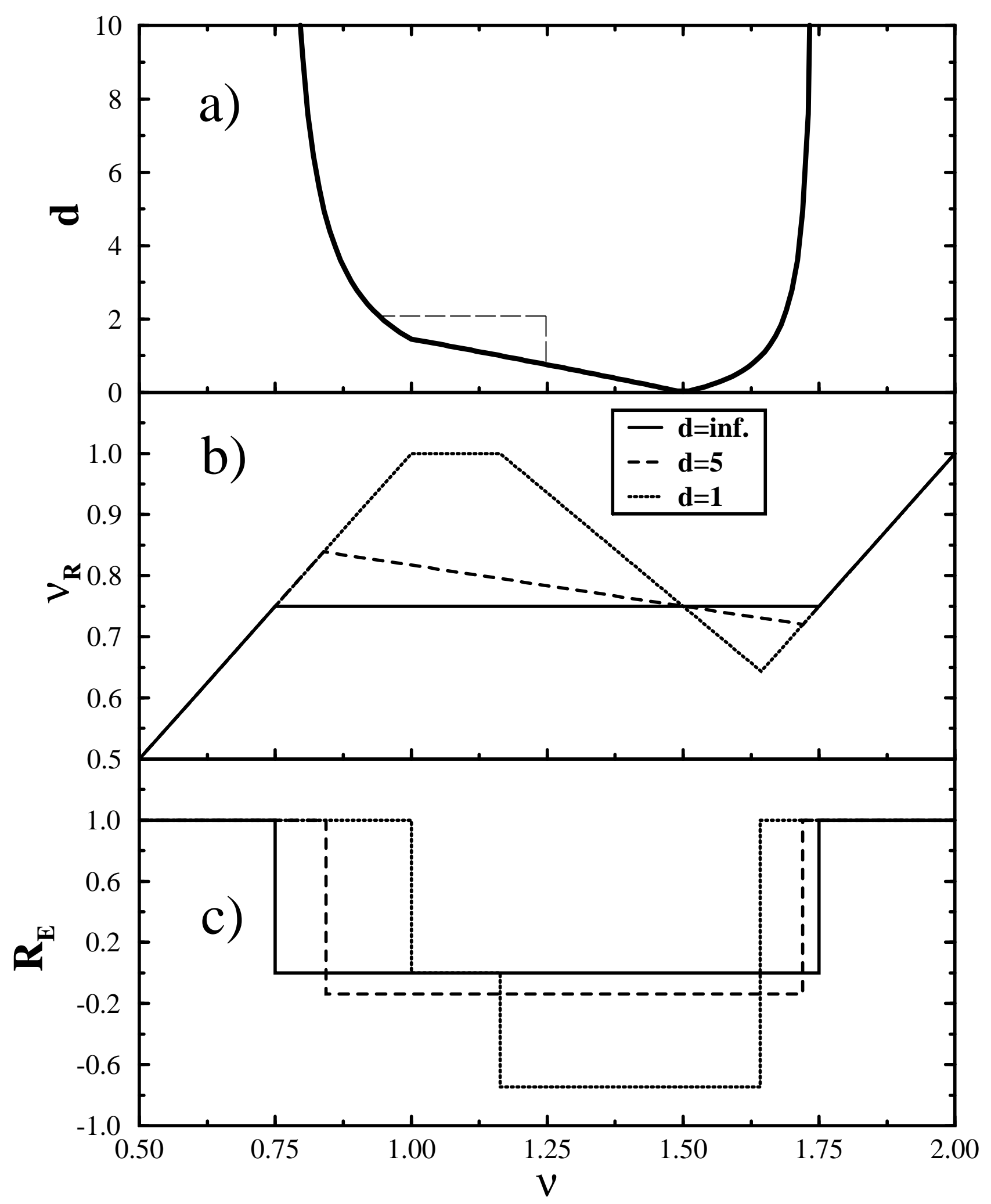




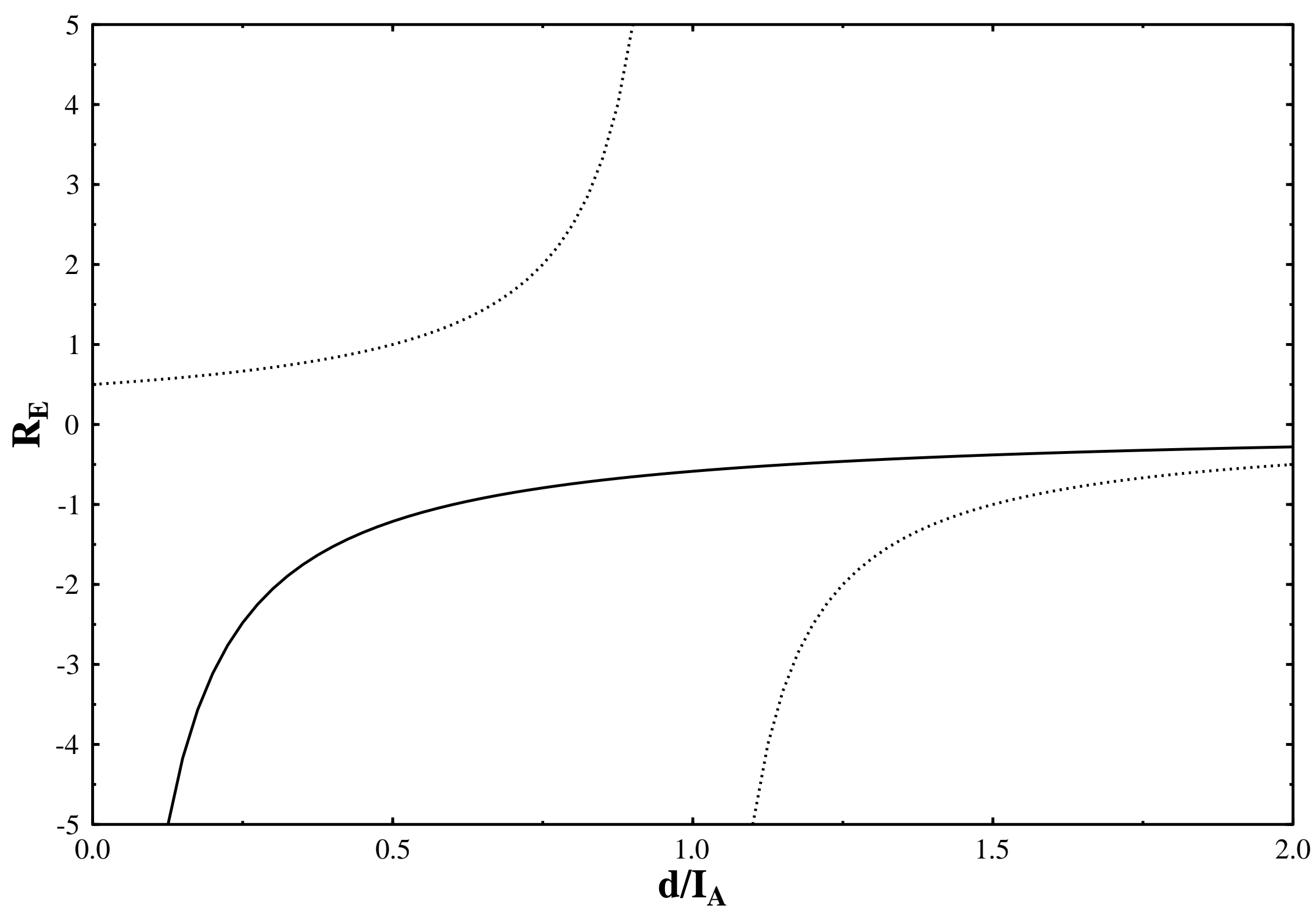




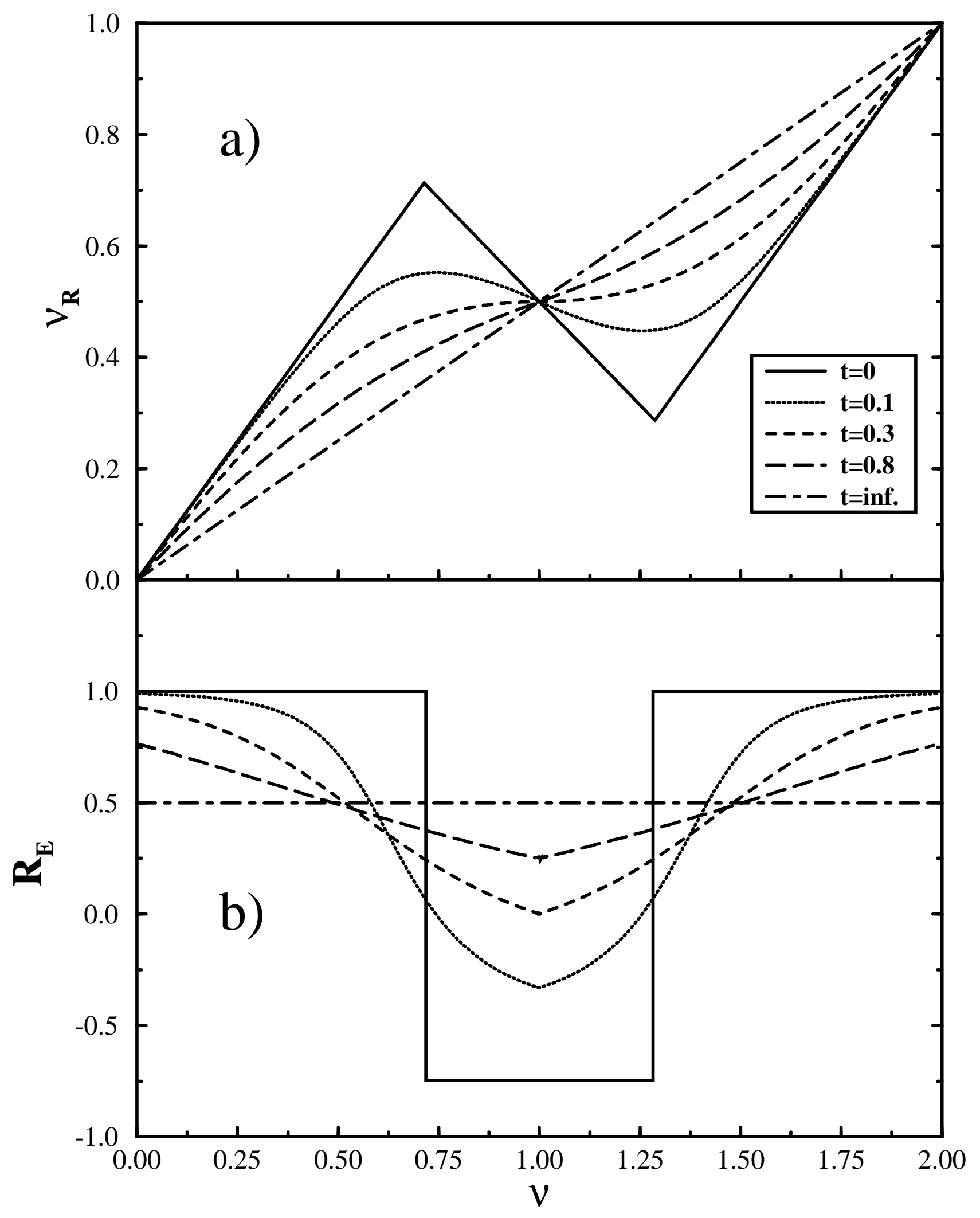

